\title{
Anomalous Voltage Noise at the Superconducting Transition of Tin Films
}

\author{
Hengsong Zhang • Fulin Zuo • \\ Massimiliano Galeazzi
}

Received: 22 July 2007 / Accepted: 15 September 2007 / Published online: 8 January 2008

(C) Springer Science+Business Media, LLC 2008

\begin{abstract}
We report voltage noise studies in the superconducting transition of thin Tin (Sn) films. Voltage noises are measured as a function of temperature and ac current. The noise spectral power $S^{1 / 2}$ strongly depends on the temperature and current, with the peak temperature in $S^{1 / 2}$ shifted down from that of $d R / d T$. Comparison with the dc noise measurement shows the $S^{1 / 2}$ is much larger with ac current than dc. $I-V$ characteristics and voltage noises are measured simultaneously to reveal the nature of the excess noises. The noise dependence on voltage strongly suggests the nature of vortex shot noise with a characteristic $\sqrt{V}$ dependence for small $V$ and reduction of $S^{1 / 2}$ due to strong correlation of vortices at large $V$.
\end{abstract}

Keywords Voltage noises $\cdot$ Superconducting transition $\cdot$ Vortex motion

PACS 74.25.Fy $\cdot 74.40 .+\mathrm{k} \cdot 74.78 . \mathrm{Db}$

\section{Introduction}

Studies of excess noises in the superconducting transition have attracted considerable attention recently in the quest to achieve superior energy resolution using superconducting transition-edge micro-calorimeter than conventional Silicon based detectors $[1,2]$. Two known noise sources are the Johnson noise and thermal fluctuations of the sensor itself [3]. Other models including flux noises and fluctuation of inhomogeneities etc. have been proposed. Experimentally, various compositional, and structural modifications have been tested to reduce the excess noises such as using Corbino disk [4], wedge, stripe structure [5] etc. However, while there has been empirical progress, the origin of the excess noises is still controversial.

H. Zhang · F. Zuo $(\bowtie) \cdot$ M. Galeazzi

Department of Physics, University of Miami, Coral Gables, FL, USA

e-mail: zuo@physics.miami.edu 
In this paper we present noise studies in an experimentally easily accessible and physically analogous classical low temperature superconductor, i.e. the metallic thin Tin films. Unlike most noise measurements where dc current or voltage sources are used, ac currents are used to probe the noise response in this experiment. Our results show qualitatively similar temperature and current dependence as in the dc case, however, with a much larger overall magnitude of the noise power.

\section{Experimental Set-Up}

Thin granular Tin films were deposited on a patterned structure on glass substrate by thermal evaporation in vacuum $\left(5 \times 10^{-5}\right.$ torr $)$ at a typical rate of $2-3 \AA / s$. A typical film has a dimension of $0.4 \mathrm{~mm} \times 1 \mathrm{~cm}$ and thickness varying from 500-1000 $\mathrm{A}$. Room temperature resistance varies from $40 \Omega$ to $100 \Omega$ and it decreases to 4-10 $\Omega$ before transition. Superconducting transition occurs at about $3.85 \mathrm{~K}$ with typical transition width of 20-40 mK. Samples are immersed in liquid helium bath and the temperature is controlled by pumping on the bath for constant temperature measurements. Temperature dependence of the voltage noise was measured while the bath is slowly pumping down or drifting up at typical rate of $1-3 \mathrm{uK} / \mathrm{s}$. The noise spectra were recorded using an SR760 spectrum analyzer with two-stage amplifications. The noise signals were first fed into a low noise transformer amplifier followed by low noise pre-amplifiers with a typical total amplification of $10^{5}$. Noise power reported here was measured at typically $1 \mathrm{kHz}$ and the excitation ac current was at $16 \mathrm{~Hz}$.

\section{Results and Discussion}

Shown in Fig. 1 is an overlay of the temperature dependence of the noise power $S^{1 / 2}$, the resistance $R$ and the derivative of the resistance $d R / d T$ during the transition at $1 \mathrm{uA}$. $d R / d T$ and $S^{1 / 2}$ are multiplied by $10^{-2}$ and $10^{10}$, respectively, to be displayed on the same scale as $R(T)$. The superconducting transition was at about $3.85 \mathrm{~K}$ and a transition width was about $20 \mathrm{mK}$.

The noise power $S^{1 / 2}$ is strongly peaked during the transition with the peak slightly shifted toward lower $T$ from that of $d R / d T$. At $1 \mathrm{uA}$, the maximum $S^{1 / 2}$ is about $0.5 \mathrm{nV} / \sqrt{\mathrm{Hz}}$.

Similar temperature dependence has been observed for different excitation currents. In general, $S^{1 / 2}$ increases with increasing $I$. To examine the current dependence of the noise power more carefully, we have carried out simultaneous measurements of voltage and $S^{1 / 2}$ at fixed temperatures. At low temperatures, the $I-V$ curve displays strong power-law dependence with $V \propto I^{\alpha}$ with $\alpha \geq 3$. Shown in Fig. 2(a) is a plot of the voltage vs. $I$ at $T=3.822 \mathrm{~K}$ with $\alpha=3.2$. $S^{1 / 2}$ increases linearly with $\sqrt{V}$, as shown in Fig. 2(b). The different symbols are for repeat measurements at the same temperature and the lines are guides for the power-law dependence.

At higher temperatures when the sample exhibits finite resistance at small $I, V$ displays linear dependence in $I$ at small current and evolves into a power-law dependence at large $I$, as shown in Fig. 3(a) in a log-log scale at $T=3.835 \mathrm{~K}$. The arrow 


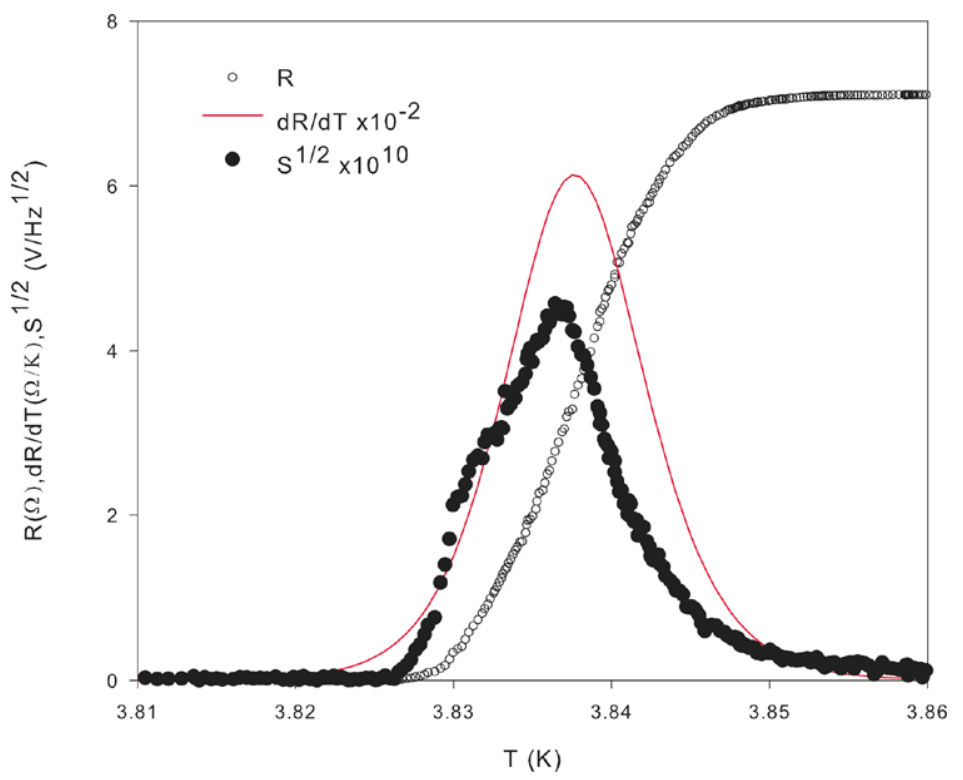

Fig. 1 (Color online) Temperature dependence of $S^{1 / 2}, d R / d T$, and $R(T)$ at $1 \mathrm{uA}$

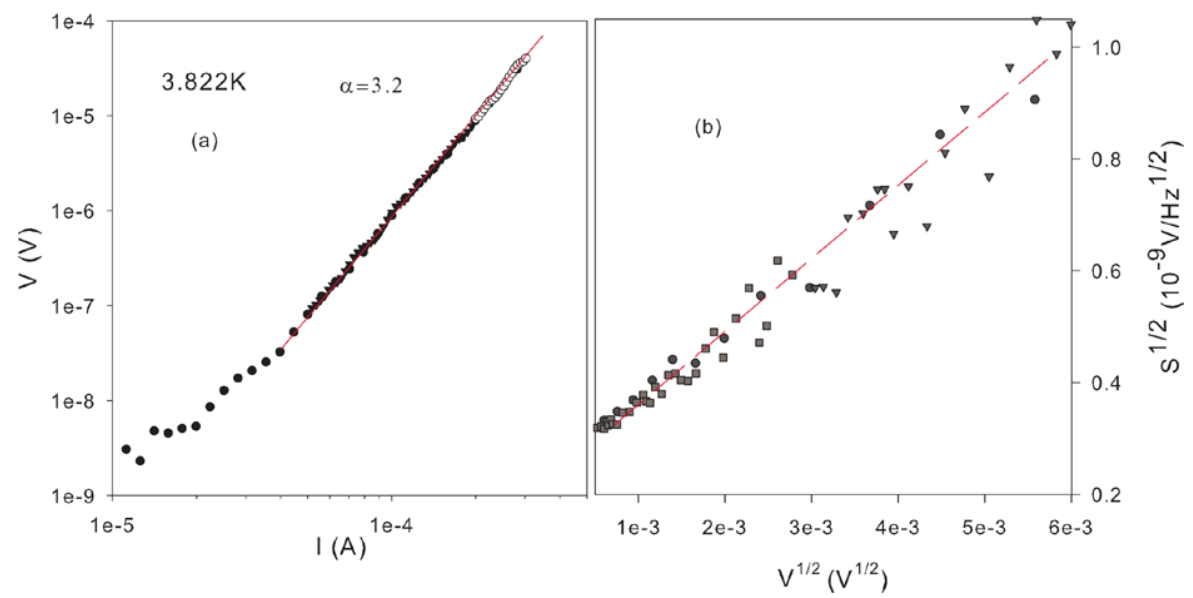

Fig. 2 (Color online) (a) Power-law dependence $V \propto I^{\alpha}$; (b) Linear dependence of $S^{1 / 2}$ vs. $V^{1 / 2}$ at $T=3.822 \mathrm{~K}$

points to a crossover from linear to power-law dependence. Figure 3(b) shows the corresponding current dependence of $S^{1 / 2}$. Unlike that at low temperatures, a peak in $S^{1 / 2}$ is observed as a function of $I$. In fact, the peak appears to occur at the same $I$ as in the crossover. For small current, $S^{1 / 2}$ is again linear with $\sqrt{V}$ as in Fig. 3(c). The open and filled symbols are for different measurements at the same temperature.

The temperature and voltage dependence of the voltage noise is of interest here. The peak noise power exceeds the thermal noise at this temperature by orders of 


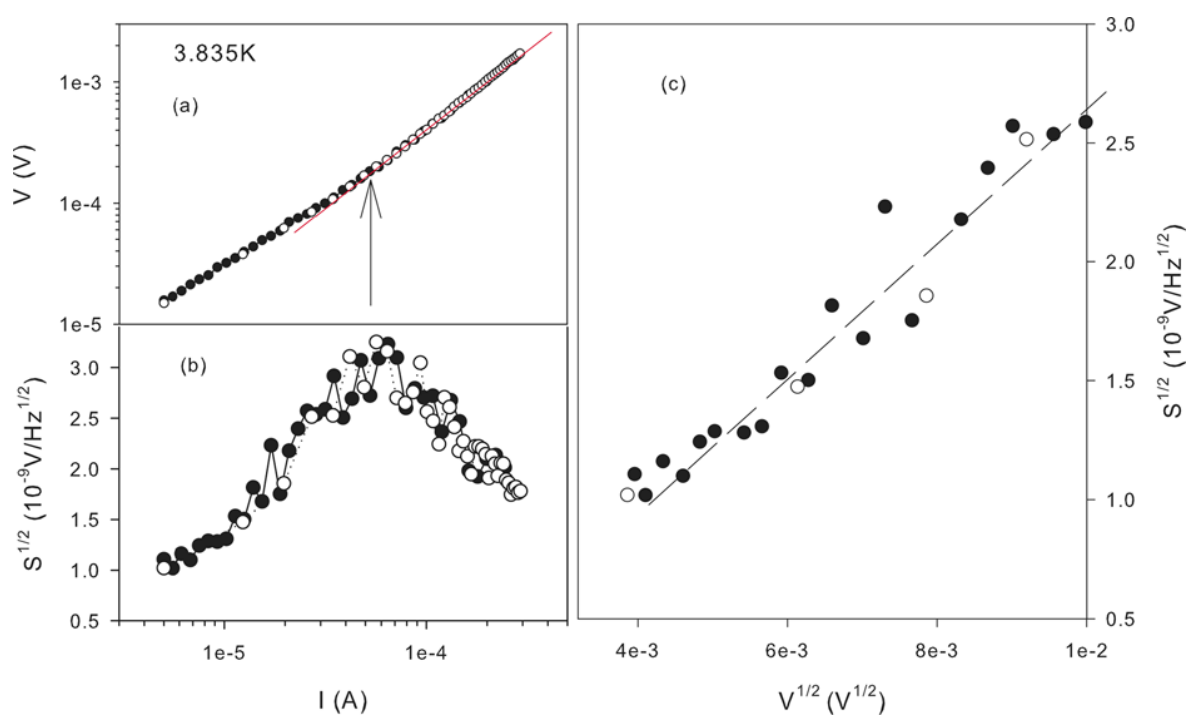

Fig. 3 (Color online) Various current, voltage and noise dependence at $T=3.835 \mathrm{~K}$

magnitude. It should be noted that the excess noise in Sn films has been reported and discussed in earlier studies [6,7]. The samples studied earlier were generally $10^{2}-10^{3}$ times more resistive than our samples, but the superconducting transitions appear very similar. The excess noise was attributed to phase-slip shot noises due to independent vortex motion.

Unlike flux-flow noise, where external magnetic field and critical current are the driving forces, the excess noises observed in the transition are due mostly to self vortices (thermally generated vortex pairs and due to field of the self current). The motion of these vortices (displacement of $l$ ) across the sample (width $w$ ) causes a change in the phase difference $\varphi$ and thus a voltage across the sample. If the vortex motion consists of independent, random phase slips of magnitude $\Delta \varphi=\frac{l}{w} 2 \pi$ during time $\Delta \tau$, the Josephson relation gives $V_{d c}=\frac{\varphi_{o}}{2 \pi} \frac{\Delta \varphi}{\Delta \tau}=\frac{\varphi_{o \Delta \varphi}}{2 \pi} r$, where $r=\frac{1}{\Delta \tau}$ is the average rate of flux motion. In analogy with the usual electrical shot noise, the noise spectral density in the case of flux motion becomes $S_{f}=2 \frac{\varphi_{o \Delta \varphi}}{2 \pi} V_{d c}$, if $f \leq \frac{1}{\tau_{p}}$ where $\tau_{p}$ is the characteristic time duration of $\Delta \varphi$.

In the case here, the linear dependence of $S_{f}^{1 / 2}$ in $\sqrt{V}$ is qualitatively consistent with the shot noise nature of the vortex motion. This is consistent with the reported data in more resistive Sn samples [6]. However, the magnitude of slope derived from $S^{1 / 2}$ vs. $V^{1 / 2}$ as in Fig. 2 and Fig. 3 is in the range of $(1.1-2.5) \times 10^{-7}$, considerably larger than the simple shot noise slope of $\sqrt{2 \varphi_{o}}=0.6 \times 10^{-7}$.

The dramatic effect of ac excitation current on the noises is interesting. Direct comparisons between dc current and ac currents indeed show a several fold (up to a factor of 10 depending on the current and temperature) of increase in the noise magnitude when ac is used while the background noise in either normal or superconducting states remains the same [8]. One possibility is the fact the ac current will naturally introduce more fluctuations in the number of self-current generated vortices or the 
number of free vortices de-paired by the driving current. Another possibility is the enhanced thermal fluctuations of the sample when an ac current is used.

\section{Conclusions}

In summary, we have carried out careful noise measurements in thin Tin films in the superconducting transition using low frequency ac current. An anomalously large noise peak is observed during the transition. The magnitude of the noise power with ac current is generally several times larger than that of a dc current. For small ac current, the noise power is seen to be linear with $\sqrt{V}$, qualitatively consistent with the model of shot noise contribution of thermally excited and current driven vortex pairs. The peak in the noise power at large current at high temperature may be indicative of correlated motion of vortices. The origin of the anomalous magnitude of the noise power is currently being investigated.

Acknowledgements This work was supported in part by NASA under Grant No. NNG05WC16G.

\section{References}

1. K.D. Irwin, Appl. Phys. Lett. 66, 1998 (1995)

2. B. Cabrera, R.M. Clarke, P. Colling, A.J. Miller, S. Nam, R.W. Romani, Appl. Phys. Lett. 73, 735 (1998)

3. H.F.C. Hoevers, A.C. Bento, M.P. Bruijn, L. Gottardi, M.A.N. Korevaar, W.A. Mels, P.A.J. de Korte, Appl. Phys. Lett. 77, 4422 (2000)

4. A. Luukanen, K.M. Kinnunen, A.K. Nuottajärvi, H.F. Hoevers, W.M. Bergmann Tiest, J.P. Pekola, Phys. Rev. Lett. 90, 238306 (2003)

5. J.N. Ullom, W.B. Doriese, G.C. Hilton, J.A. Beall, S. Deiker, W.D. Duncan, L. Ferreira, K.D. Irwin, C.D. Reintsema, L.R. Vale, Appl. Phys. Lett. 84, 4206 (2004)

6. R.F. Voss, C.M. Knoedler, P.M. Horn, Phys. Rev. Lett. 45, 1523 (1980)

7. C.M. Knoedler, R.F. Voss, Phys. Rev. B 26, 449 (1982)

8. H. Zhang, F. Zuo, to be published 